\title{
50 years of screening in the Nordic countries: quantifying the effects on cervical cancer incidence
}

\author{
S Vaccarella ${ }^{*}$, , S Franceschi ${ }^{1}$, G Engholm ${ }^{2}$, S Lönnberg ${ }^{3}$, S Khan $^{4}$ and F Bray ${ }^{1}$ \\ ${ }^{1}$ International Agency for Research on Cancer, 150 cours Albert Thomas, 69372 Lyon cedex 08, France; ${ }^{2}$ Department of \\ Documentation \& Quality, Danish Cancer Society, Strandboulevarden 49, 2100 Copenhagen, Denmark; ${ }^{3}$ Cancer Registry of \\ Norway, P.O. box 5313 Majorstuen, Oslo N-0304, Norway and ${ }^{4}$ Swedish Cancer Registry, National Board of Health and Welfare, \\ S-106 30 Stockholm, Sweden
}

Background: Nordic countries' data offer a unique possibility to evaluate the long-term benefit of cervical cancer screening in a context of increasing risk of human papillomavirus infection.

Methods: Ad hoc-refined age-period-cohort models were applied to the last 50-year incidence data from Denmark, Finland, Norway and Sweden to project expected cervical cancer cases in a no-screening scenario.

Results: In the absence of screening, projected incidence rates for 2006-2010 in Nordic countries would have been between 3 and 5 times higher than observed rates. Over 60000 cases or between 41 and $49 \%$ of the expected cases of cervical cancer may have been prevented by the introduction of screening in the late $1960 \mathrm{~s}$ and early $1970 \mathrm{~s}$.

Conclusions: Our study suggests that screening programmes might have prevented a HPV-driven epidemic of cervical cancer in Nordic countries. According to extrapolations from cohort effects, cervical cancer incidence rates in the Nordic countries would have been otherwise comparable to the highest incidence rates currently detected in low-income countries.

Cervical cancer has become a relatively rare disease in many high-income countries (Bray et al, 2012). Although it is widely recognised that cervical cancer incidence and mortality rates have dropped substantially after the implementation of high-coverage high-quality screening programmes (Laara et al, 1987; Coleman et al, 1993; Hakama and Hristova, 1997; Hristova and Hakama, 1997; Sasieni and Adams, 1999; Sasieni et al, 2003; Peto et al, 2004; Zappa et al, 2004; Bray et al, 2005; IARC, 2005; Anttila and Nieminen, 2007; Curado et al, 2007; Andrae et al, 2008; Vaccarella et al, 2013), estimation of what would have happened in the absence of screening are difficult.

In previous age-period-cohort (APC) analyses (Bray et al, 2005; Vaccarella et al, 2013), we were able to distinguish two major factors acting on cervical cancer: (1) period effect, that is linked to the increasing availability of adequate screening programmes and deflects downward incidence trends in targeted age groups; and (2) cohort effect, that reflects changes in risk factors across different birth cohorts, notably the increase in the risk of human papillomavirus (HPV) infection, the necessary cause of cervical cancer (IARC, 2005). Indirect evidence suggests that HPV infection have become more prevalent since the 1960s in a number of high-income countries (Simms and Fairley, 1997; Dillner, 2000; Peto et al, 2004), mainly due to changes in sexual habits.

The primary objective of the present analysis is to estimate the long-term impact of screening on cervical cancer in four Nordic countries. These countries offer a unique opportunity on account of the availability of high-quality and long-term cancer statistics and screening programmes. In the present analysis, we, therefore, estimated the number of cervical cancer cases that screening has probably prevented in four Nordic countries between 1961 and

*Correspondence: Dr S Vaccarella; E-mail: vaccarella@iarc.fr

Received 5 January 2014; revised 22 May 2014; accepted 4 June 2014; published online 3 July 2014

(c) 2014 Cancer Research UK. All rights reserved 0007-0920/14 
2010 comparing observed incidence rates with those expected in a hypothetical no-screening scenario.

\section{MATERIALS AND METHODS}

Data sources. National data on incident cases of invasive cervical cancer as well as population data were obtained from NORDCAN. The data sources and methods are described in detail elsewhere (Engholm et al, 2010). In brief, the source of data is based on the core dataset from NORDCAN (www.ancr.nu), a database with comparable and timely high-quality data on cancer incidence and mortality in the Nordic countries delivered from the cancer registries. For the purpose of the present analyses, we did not distinguish between squamous cell carcinoma and adenocarcinoma of the cervix. Furthermore, it was not possible to analyse microinvasive cancers separately, as the Nordcan database does not collect this information. The time span of observations was 55 years for Denmark, Finland, Norway and 50 years for Sweden. Analyses were restricted to women aged 30-74 years (Table 1).

Ethical approval was not required as this study did not involve direct contact with patients or personal identifiers.

\section{Statistical analyses}

APC models. Age-standardised incidence rates (ASRs) per 100000 person-years were calculated by the direct standardisation method, using the World standard population as a reference. We obtained birth cohorts by subtracting age (midpoint of 5-year age band) from the central year of 5-year calendar period of diagnosis.

We used APC models to summarise time trends in terms of age, cohort and period effects (Clayton and Schifflers, 1987a, b; Holford, 1998).

To obtain stable estimates using APC analysis, we grouped age and period in 5-year classes. For each 5-year age-class and 5-year period of diagnosis, the number of events and person-years corresponded to $5 \times 5$-year subsets of a Lexis diagram.
We circumvented the non-identifiability problem of APC models by taking advantage of the consistent relationship between age and cervical cancer incidence. Incidence rates of cervical cancer are expected to be approximately constant after age 45 years. In unscreened populations, incidence rates increase up to $\sim 45$ years of age and then flatten (Plummer et al, 2012). Conversely, in screened populations, incidence rates flatten at $\sim 35$ years of age, that is, when the beneficial effect of screening, that typically targets women aged 25 years or older, starts to manifests. We, therefore, constrained incidence rates to be equal at ages 45-49 and 65-69, thus enabling the estimation of a unique set of parameters for the age, period and cohort effects.

Projected incidence rates and cervical cancer cases in a no-screening scenario. We assumed that significant declines in period effects estimated by the APC models in Nordic countries were owing to the effects of cervical cancer screening. The projected ASRs based on the world standard population and corresponding number of incident cervical cancer cases were thus extrapolated assuming the existence of age and cohort effects, but imposing a hypothetical lack of period effect. Join-point regression was used to identify time points where significant declines in the slope of period trends occurred. The first significant join-points were identified to correspond to period 1961-1965 for Denmark and Finland, 1971-1975 for Norway and 1966-1970 for Sweden. In a no-screening scenario, period effects subsequent to these periods were set to be constant, instead of declining. Non-significant fluctuations for periods preceding significant declines were, however, maintained unchanged. The cumulative number of cervical cancer cases that have been prevented by screening over the available time span (1961-2010) was computed as the difference between the projected estimates based on APC model and cumulatively observed cases from NORDCAN (Engholm et al, 2010).

Screening activities. In Denmark, cervical screening started in 1962. Regional screening programmes covered $\sim 40 \%$ of the population in 1967, and national roll-out was completed in 1996.

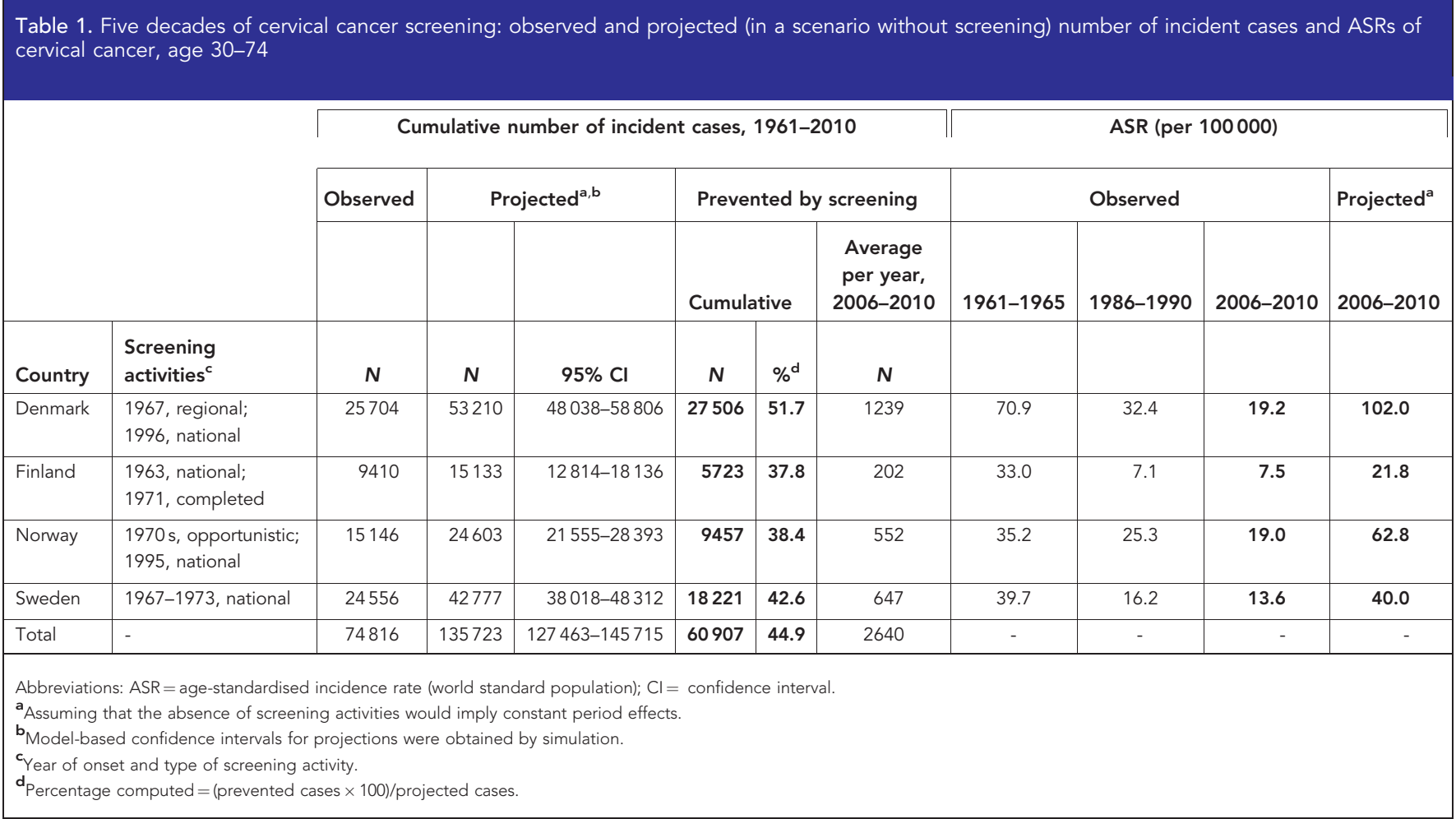


In Finland, cervical screening started in 1961, and a national population-based screening programme was established in 1963 and completed in 1971. In Norway, cervical screening started in 1959 in one county and opportunistic screening activities substantially increased during the 1970s. A national centralised screening programme based on a combination of opportunistic and organised activities was implemented in 1995. In Sweden, cervical screening started in 1964 and it was expanded to a national programme between 1967 and 1973 (Anttila et al, 2004).

\section{RESULTS}

According to our projections of a no-screening scenario, cervical cancer incidence rates between 1961 and 2010 would have increased in Denmark and Norway and remained stable in Sweden. In Finland, declines would have been substantially smaller than those observed. Projected incidence rates in 2006-2010 would have been $\sim$ threefold higher than those observed in Finland, Norway and Sweden and fivefold higher in Denmark. Cervical cancer incidence rates in Denmark and Norway in 2006-2010 would have, therefore, been 102 (observed: 19) and 63 (observed: 19) per 100000 , respectively (Table 1 and Figure 1), that is, comparable to the highest rates observed in sub-Saharan countries (Ferlay et al, 2010). Rates in Finland and Sweden would have been 22 (observed: 8) and 40 (observed: 14) per 100000 , respectively (Table 1 and Figure 1), that is, comparable to the highest rates observed in Eastern Europe, Latin America and Asia (Ferlay et al, 2010). Table 1 also shows the projections relative to the average yearly number of cervical cancer cases that have been prevented by screening in 2006-2010 (from 202 in Finland to 1239 in Denmark). Overall in the Nordic countries, over 60000 cases or between
41 and $49 \%$ of the expected cases of cervical cancer may have been prevented by the introduction of screening in the late 1960s and early 1970 s.

\section{DISCUSSION}

Our present projections are based on the assumption that the observed decline of the period effects can be attributed to screening and that a counterfactual no-screening scenario can be obtained by using the age and cohort effects from our APC model but enforcing the period effect to be constant over time.

While period effects may not be exclusively attributable to screening, other explanations are unlikely to have been major contributors in Nordic countries. Previous analyses in Australia and the UK have used a similar assumption (Sasieni and Adams, 1999; Taylor et al, 2001). High parity, for instance, is a risk factor for cervical cancer (International Collaboration of Epidemiological Studies of Cervical Cancer, 2006) and fertility rates in Nordic countries had declined during the 1960s. However, they had remained approximately constant since the mid 1970s (The World Bank Group, 2010). In addition, changes in fertility rates tend to occur progressively across successive cohorts of women, and the possible impact on cervical cancer rates would be expected to mainly manifest itself as a cohort, rather than period, effect.

Further support to the importance of screening is provided by the comparison with the findings of similar APC models in Eastern European countries (Arbyn et al, 2011; Vaccarella et al, 2013). Nordic countries and Eastern European countries showed similar increases in cervical cancer incidence rates among the youngest birth cohorts, most likely due to the progressive increase in the risk of HPV exposure. However, Eastern European countries showed no favourable period effects, likely reflecting the lack of adequate
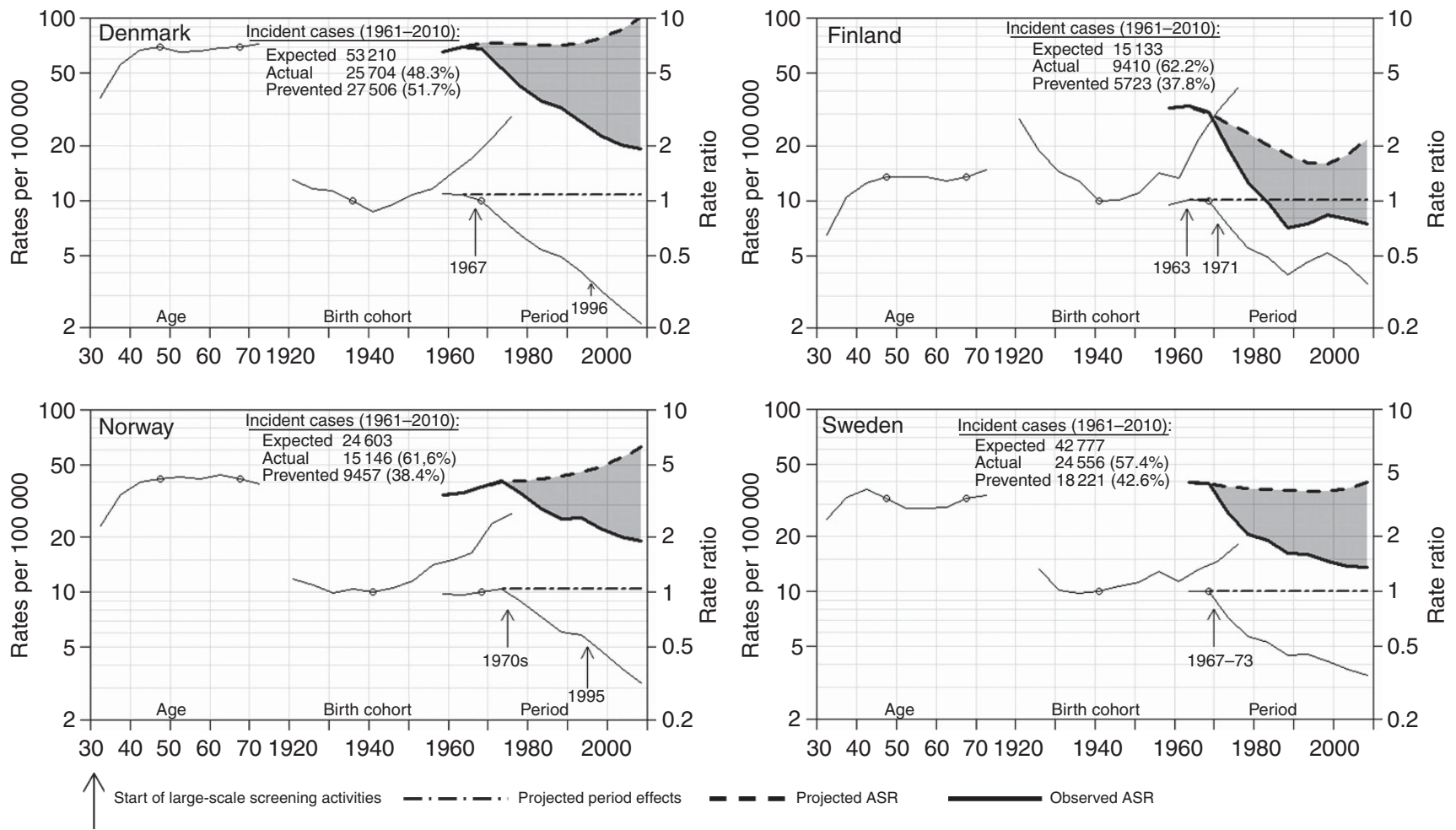

Figure 1. Effect of age, period and cohort on observed and projected ASRs of incident cervical cancer. Age-standardised rates (ASRs) are plotted by period. Age effects and ASRs are shown on a rate per 100000 scale; cohort and period effects are on a relative risk scale. Reference points for period and cohort rate ratios are marked. Under a scenario without screening activities, period effects are forced to assume a constant value over time (dot-dashed lines). The projected ASRs corresponding to period effects constant over time, but age and cohort effects as estimated by the model, are also shown (dashed thick lines). 
screening activities, and resulting in upward or stable incidence rates.

The estimates of cohort effects assume a particular importance as, under the assumption of no period effects, they shape the projected trends. For instance, the pronounced U-shaped birthcohort effect observed in Finland, with a marked decline from generations of women born between 1920 and 1940 and a subsequent increase, causes a similar shaped pattern of the projected rates: an initial decline from the mid 1960s to the mid 1990s followed by an increase. In other Nordic countries the U-shape pattern of the cohort effects, in particular the decline in generations of women born before the 1940s, is less pronounced, so that no decline is observed in the projected rates but rather stable or increasing trends. This might explain why the population impact of screening in Finland is, although important, similar or lower than that found for other Nordic countries, a result that contrasts with previous reports (Hakama and Hristova, 1997). Finland has been the country where one of the first indications of the magnitude of the impact of cervical cancer screening was observed in a cohort study (Hakama and Rasanen-Virtanen, 1976). Although there is no clear explanation, changes in lifestyle habits, particularly in sexual behaviour, or in fertility rates may be possible reasons for the declining cohort effects in older generations in Finland. Favourable incidence and mortality rates of cervical cancer prior to the introduction, or in absence, of screening have also been observed in other countries, including the UK (Peto et al, 2004; Vaccarella et al, 2013). We cannot, however, exclude that a residual effect of screening could be visible as a cohort, instead of period effect, and explain the declining risk in women born between 1920s and 1940s: in that case, the population impact of screening in Finland would be underestimated.

Strengths of our APC analysis include the availability of high-quality population-based cancer registry data over a 50 -year period, that is, prior to the establishment of screening programmes. This allows the estimation of the 'cumulative' impact of screening on the population even when detailed information on screening coverage, organisational aspects and time changes, for example, from opportunistic $v s$ organised screening are not available. We might not exclude, however, that the initial impact of screening may be partly obscured by the early detection of microinvasive cancers.

Contrary to the UK (Sasieni and Adams, 1999; Peto et al, 2004), favourable period effects had started manifesting themselves in Nordic countries since large screening campaigns had been established even if not yet as national organised programmes. Nordic countries are smaller and less heterogeneous populations than the British population and may, therefore, have achieved reasonably high-quality cervical cancer screening more rapidly.

In conclusion, our study suggests that screening programmes and treatment of screen-detected cervical lesions might have prevented over 60000 cases of invasive cervical cancer in the Nordic countries, that is, nearly half of the cases that would have been expected in the lack of screening. The beneficial impact of screening steadily increased over time. Cervical cancer would not be a rare disease in Nordic countries and in other high-income countries had it not been for the substantial investments in screening. To continue observing favourable trends, screening programmes should be continued and improved, for example, shifting from cytology-based to HPV testing-base primary screening (Ronco et al, 2013), and backed by the prevention of HPV infection through vaccination (Crosbie et al, 2013).

\section{CONFLICT OF INTEREST}

The authors declare no conflict of interest.

\section{REFERENCES}

Andrae B, Kemetli L, Sparen P, Silfverdal L, Strander B, Ryd W, Dillner J, Tornberg S (2008) Screening-preventable cervical cancer risks: evidence from a nationwide audit in Sweden. J Natl Cancer Inst 100: 622-629.

Anttila A, Nieminen P (2007) Cervical cancer screening programme in Finland with an example on implementing alternative screening methods. Coll Antropol 31(Suppl 2): 17-22.

Anttila A, Ronco G, Clifford G, Bray F, Hakama M, Arbyn M, Weiderpass E (2004) Cervical cancer screening programmes and policies in 18 European countries. Br J Cancer 91: 935-941.

Arbyn M, Antoine J, Magi M, Smailyte G, Stengrevics A, Suteu O, Valerianova Z, Bray F, Weiderpass E (2011) Trends in cervical cancer incidence and mortality in the Baltic countries, Bulgaria and Romania. Int J Cancer 128: 1899-1907.

Bray F, Jemal A, Grey N, Ferlay J, Forman D (2012) Global cancer transitions according to the Human Development Index (2008-2030): a population-based study. Lancet Oncol 13: 790-801.

Bray F, Loos AH, McCarron P, Weiderpass E, Arbyn M, Moller H, Hakama M, Parkin DM (2005) Trends in cervical squamous cell carcinoma incidence in 13 European countries: changing risk and the effects of screening. Cancer Epidemiol Biomarkers Prev 14: 677-686.

Clayton D, Schifflers E (1987a) Models for temporal variation in cancer rates. I: Age-period and age-cohort models. Stat Med 6: 449-467.

Clayton D, Schifflers E (1987b) Models for temporal variation in cancer rates. II: age-period-cohort models. Stat Med 6: 469-481.

Coleman MP, Esteve J, Damiecki P, Arslan A, Renard H (1993) Trends in Cancer Incidence and Mortality. International Agency for Research on Cancer: Lyon, France.

Crosbie EJ, Einstein MH, Franceschi S, Kitchener HC (2013) Human papillomavirus and cervical cancer. Lancet 382: 889-899.

Curado MP, Edwards B, Shin HR, Storm H, Ferlay J, Heanue M, Boyle P (2007) Cancer Incidence in Five Continents Volume IX. International Agency for Research on Cancer: Lyon, France, IARC Scientific Publications No. 160.

Dillner J (2000) Trends over time in the incidence of cervical neoplasia in comparison to trends over time in human papillomavirus infection. J Clin Virol 19: 7-23.

Engholm G, Ferlay J, Christensen N, Bray F, Gjerstorff ML, Klint A, Kotlum JE, Olafsdottir E, Pukkala E, Storm HH (2010) NORDCAN-a Nordic tool for cancer information, planning, quality control and research. Acta Oncol 49: 725-736.

Ferlay J, Shin HR, Bray F, Forman D, Mathers C, Parkin DM (2010) Estimates of worldwide burden of cancer in 2008: GLOBOCAN 2008. Int J Cancer 127: 2893-2917.

Hakama M, Hristova L (1997) Effect of screening in the Nordic cancer control up to the year 2017. Acta Oncol 36: 119-128.

Hakama M, Rasanen-Virtanen U (1976) Effect of a mass screening program on the risk of cervical cancer. Am J Epidemiol 103: 512-517.

Holford TR. Age-period-cohort analysis. In Armitage P, Colton T (eds) Encyclopedia of Biostatistics. John Wiley \& Sons: Chichester, UK (1998) pp 82-99.

Hristova L, Hakama M (1997) Effect of screening for cancer in the Nordic countries on deaths, cost and quality of life up to the year 2017. Acta Oncol 36(Suppl 9): 1-60.

IARC (2005) IARC Handbooks of Cancer Prevention Volume 10: Cervix Cancer Screening. IARC Press: Lyon, France.

International Collaboration of Epidemiological Studies of Cervical Cancer (2006) Cervical carcinoma and reproductive factors: collaborative reanalysis of individual data on 16563 women with cervical carcinoma and 33542 women without cervical carcinoma from 25 epidemiological studies. Int J Cancer 119: 1108-1124.

Laara E, Day NE, Hakama M (1987) Trends in mortality from cervical cancer in the Nordic countries: association with organised screening programmes. Lancet 1: 1247-1249.

Peto J, Gilham C, Fletcher O, Matthews FE (2004) The cervical cancer epidemic that screening has prevented in the UK. Lancet 364: 249-256.

Plummer M, Peto J, Franceschi S (2012) Time since first sexual intercourse and the risk of cervical cancer. Int J Cancer 130: 2638-2644.

Ronco G, Dillner J, Elfstrom KM, Tunesi S, Snijders PJ, Arbyn M, Kitchener H, Segnan N, Gilham C, Giorgi-Rossi P, Berkhof J, Peto J, Meijer CJ (2013) Efficacy of HPV-based screening for prevention of 
invasive cervical cancer: follow-up of four European randomised controlled trials. Lancet 383: 524-532.

Sasieni P, Adams J (1999) Effect of screening on cervical cancer mortality in England and Wales: analysis of trends with an age period cohort model. BMJ 318: 1244-1245.

Sasieni P, Adams J, Cuzick J (2003) Benefit of cervical screening at different ages: evidence from the UK audit of screening histories. Br J Cancer 89: $88-93$.

Simms I, Fairley CK (1997) Epidemiology of genital warts in England and Wales: 1971 to 1994. Genitourin Med 73: 365-367.

Taylor RJ, Morrell SL, Mamoon HA, Wain GV (2001) Effects of screening on cervical cancer incidence and mortality in New South Wales implied by influences of period of diagnosis and birth cohort. J Epidemiol Community Health 55: 782-788.

The World Bank Group (2010) The World Bank. http://web.worldbank.org/.

Vaccarella S, Lortet-Tieulent J, Plummer M, Franceschi S, Bray F (2013)

Worldwide trends in cervical cancer incidence: Impact of screening against changes in disease risk factors. Eur J Cancer 49: 3262-3273.

Zappa M, Visioli CB, Ciatto S, Iossa A, Paci E, Sasieni P (2004) Lower protection of cytological screening for adenocarcinomas and shorter protection for younger women: the results of a case-control study in Florence. $\mathrm{Br} J$ Cancer 90: 1784-1786. 